\title{
三维点云模型特征张量描述符的构造及自相似性分析
}

\author{
胡海龙 ${ }^{1,2)}$, 李重 ${ }^{3)^{*}}$, 秦胜伟1)，马利庄 ${ }^{4)}$ \\ 1) (浙江理工大学机械与自动控制学院 杭州 310018) \\ 2) (浙江农林大学理学院 杭州 311300) \\ 3) (浙江理工大学理学院 杭州 310018) \\ ${ }^{4)}$ (上海交通大学电子信息与电气工程学院 上海 200240) \\ (lizhong@zstu.edu.cn)
}

\begin{abstract}
摘 要: 三维模型局部自相似性是物体形状分析中的一个基本问题, 其中, 局部形状描述符的构建对自相似性分析 的最终结果至关重要. 针对此问题, 提出了一种基于张量融合特征描述符的自相似性分析方法. 首先利用相关面和 反向点对点云模型进行形状直径函数(shape diameter function, SDF)的近似计算; 然后利用谱聚类对模型进行过分割 成模型子块, 由 $K$ 近邻 $(K$-nearest neighbor, $K \mathrm{NN})$ 邻域点的 SDF 、形状指数(shape index, SI)和高斯曲率(Gauss curvature, GS)矩阵构造三维特征张量; 最后利用张量范数构造映射得到形状描述符, 并定义相似性度量分析模型子块之间的 自相似性. 对几种最新的方法(包括部分匹配和显著性检测)进行了实验，无论是直观视觉效果，还是相似性测度和相 对误差上的评价指标, 结果均表明, 该方法可有效地对形状进行描述，提高了点云模型相似子块的识别精度.
\end{abstract}

关键词: 自相似性; 三阶张量; 形状分析; 点云模型

中图法分类号: TP391.41 DOI: 10.3724/SP.J.1089.2021.18542

\section{Construction of Feature Tensor Descriptor and Self-Similarity Analysis for 3D Point Cloud Models}

\author{
Hu Hailong ${ }^{1,2)}$, Li Zhong ${ }^{3)^{*}}$, Qin Shengwei ${ }^{1)}$, and Ma Lizhuang ${ }^{4)}$ \\ ${ }^{1)}$ (Faculty of Mechanical Engineering \& Automation, Zhejiang Sci-Tech University, Hangzhou 310018) \\ 2) (School of Science, Zhejiang A\&F University, Hangzhou 311300) \\ ${ }^{3)}$ (School of Science, Zhejiang Sci-Tech University, Hangzhou 310018) \\ ${ }^{4)}$ (College of Electronic Information and Electrical Engineering, Shanghai Jiao Tong University, Shanghai 200240)
}

\begin{abstract}
Local self-similarity of 3D model is a fundamental problem in the shape analysis. The construction of a local shape descriptor is very important to the final result of self-similarity analysis. To solve this problem, a self-similarity analysis method based on the tensor fusion feature descriptor is proposed. Firstly, the shape diameter function (SDF) of a point cloud model is approximately calculated by using relevant facets and antipodal points. Then, spectral clustering is used to segment the model into sub-blocks, and the three-dimensional feature tensor is constructed from the SDF, shape index (SI) and Gauss curvature (GS) matrix of $K \mathrm{NN}$ neighborhood points. Finally, the shape descriptor is obtained by constructing the mapping with the tensor norm, and then the similarity measure is defined and the self-similarity between the sub-blocks of the model is analyzed. Several state-of-the-art methods (including partial matching and saliency detection) are
\end{abstract}

收稿日期：2020-08-04; 修回日期：2020-09-26. 基金项目：国家自然科学基金(11671009); 浙江省自然科学基金(LY19F010014, LZ19A010002). 胡海龙(1980一), 男, 博士, 讲师, 主要研究方向为计算机图形学、数字几何处理; 李重(1975一), 男, 博士, 教授, 博士生导师, CCF 会员, 论文通讯作者, 主要研究方向为计算机图形学、数字几何处理; 秦胜伟(1989-), 男, 博士, 讲师, 主要研究 方向为计算机图形学、数字几何处理; 马利庄(1963-), 男, 博士, 教授, 博士生导师, 主要研究方向为计算机图形图像处理、多媒体 与虚拟现实. 
tested. In terms of not only the visual effect, but also the similarity measure and the relative errors, the results show that this method can effectively describe the shape and improves the recognition accuracy of similar sub-blocks of a point cloud model.

Key words: self-similarity; three-order tensor; shape analysis; point cloud models

三维模型的形状分析是实现三维模型检索、压 缩、多分辨率建模等的关键技术, 已成为计算机图 形学和数字几何处理领域的一个热门课题 ${ }^{[1]}$. 对象 的局部自相似性是指给定模型中一个区域与其他 区域之间的相似程度. 由于它能够描述模型表面 局部区域之间的相似性和关联度, 因此在三维模 型的局部匹配、模型识别和形状对齐等方面得到了 广泛的应用 ${ }^{[2]}$.

\section{1 相关工作}

对于三维模型的局部自相似性分析，通常是 构造其局部形状描述符，然后对模型的不同子区 域进行相似性分析. 相关工作包括基于局部形状 描述符的局部匹配和基于局部形状描述符的显著 性检测等局部形状分析方法.

\section{1 基于局部形状描述符的部分匹配}

Gal 等 ${ }^{[3]}$ 介绍了一种对三角网格表示的曲面进 行局部匹配的方法, 它可以匹配数值和拓扑上不 同但相似的表面区域. Arbel 等 ${ }^{[4]}$ 使用最近邻域的 属性提供三维形状的部分匹配关系，但由于模型 拓扑噪声大, 变形量大, 这些情况很难确定最近邻 点, 因此该方法不适用于拓扑噪声大, 变形量大的 模型. Vock 等 ${ }^{[5]}$ 提出了一种基于模板的三维点云模 型局部匹配和姿态估计方法, 其速度快, 但当模型 具有平面或低密度点云时, 会产生不正确的边缘 检测, 且自相似性较差. Itskovich 等 ${ }^{[6]}$ 提出了一种 基于概率框架的自相似性分析方法, 结合了合成 分割块和特征点的相似性, 但是如果分割效果不 明显，则无法检测到相似的子块. Gatzke 等 ${ }^{[7]}$ 使用 曲率函数表示不同的局部邻域点, 然后比较曲率 函数对的差异, 得到 2 个区域的相似性. Koenderink 等 ${ }^{[8]}$ 构造了一个用于局部形状分析的形状指数 (shape index, SI), 可用于检测物体的凸凹程度. 它 们的公式也与曲率估计有关, 如果曲率估计误差 存在, 则会影响局部形状分析结果. Shapira 等 ${ }^{[9]}$ 人形状直径函数(shape diameter function, SDF)的 概念进行形状分析, 对于曲面网格上的一个点, 使 用围绕其向内法向中心的圆雉体, 并将该圆雉体
内的多条射线发射到网格模型的另一侧，然后将 其 SDF 定义为距离所有长度中值在一个标准偏差 内的所有射线长度的加权平均值. 然而, 这种方法 的主要问题是射线和网格模型之间的交点不能应 用于三维点云模型.

\section{2 基于局部形状描述符的显著性检测}

Wang 等 ${ }^{[10]}$ 提出了一种能反映局部和全局特性 的基于曲率的形状描述符，并将其应用于物体的 显著性检测. 从全局匹配的角度来看, Song 等 ${ }^{[11]}$ 利 用对数拉普拉斯谱构造形状描述符, 并对三维网 格模型进行了显著性检测; 但其有时不能有效地 识别模型相同部分的特征. $\mathrm{Wu}$ 等 ${ }^{[12]}$ 通过使用局部 对比度和全局稀有度的线性组合引人了形状描述 符, 该方法具有旋转不变性, 但由于多尺度特征描 述符的计算, 其时间复杂度较高. Lee 等 ${ }^{[13]}$ 在加权 平均曲率上使用中心边界算子定义形状描述符, 然后将其应用于检测三维网格模型上的显著性.

\section{3 局部形状分析的其他相关工作}

Bronstein 等 ${ }^{[14]}$ 提出了一种基于模型表面热扩 散函数的形状不变热核特征(heat kernel signature, $\mathrm{HKS}$ ), 它在等距和等体积变换下是不变的，在时 域上具有相对较低的特征复杂度、较少的信息损失 和多尺度特征, 但计算复杂度也较高. $\mathrm{Du}$ 等 $^{[15]}$ 提 出了一种利用测地圆盘谱构造局部形状描述符的 方法, 分析了任意边界的三维模型的形状相似性, 但其不适用于局部光滑形状变化较小的物体. Maximo 等 ${ }^{[16]}$ 通过构造基于高度场的局部形状描 述符, 对三维对象进行局部相似性分析. 虽然描述 符是旋转不变的, 但高度场与局部切平面构造有 关, 使得计算更加复杂, 可能存在误差.

通常, 上述方法主要是基于曲率或其他单个 特征构造形状描述符进行对象的形状分析. 在计 算机视觉和图像处理中, 部分匹配、相似性检测和 目标识别任务已经有了一些特征组合方法 ${ }^{[17]}$, 但 这些方法很难直接用于点云模型的三维目标对象. 如何将各种特征形式结合起来进行局部匹配, 仍 然是三维点云模型自相似性分析的一个挑战.

本文提出了一种三维点云模型自相似性分析 方法. 其主要贡献是：(1) 根据点云模型的相关面 
和反向点，提出了一种计算方便、有效的点云模型 SDF 的近似方法; (2) 结合高斯曲率(Gauss curvature, GS), SI 和 SDF 等多种特征, 利用张量范数及 其分量范数对给定参考块进行相似块集搜索; (3) 利用张量范数及其分量范数构造局部形状描述符, 不仅可以确定三维模型的相似部分，而且可以可 视化三维模型，反映物体的局部形状分布. 该描述 符由多个特征组成, 能够反映物体的几何性质, 得 到满意的自相似性分析结果.

\section{2 本文算法}

\section{1 算法概述}

在介绍本文算法概述之前，首先给出一些概 念描述. 三维模型有不同的表示形式. 点云是某 些坐标系中的一组数据点. 在三维坐标系中, 这 些点由坐标 $(x, y, z)$ 定义, 通常用于表示对象的 外表面, 称为点云模型. 本文方法将对三维点云 模型进行自相似性分析. 对于三维模型的形状分 析，早期的方法是基于曲率或其他单个特征构造
形状描述符. 首先，本文扩展了通过特征向量 ${ }^{[18]}$ 和特征矩阵 ${ }^{[19]}$ 进行相似性分析的方法，将不同的 特征整合成张量形式, 以获得三维点云模型的自 相似性.

本文的形状描述符构造和自相似性分析的主 要过程如下. 首先, 对点云模型上一点的法向量和 曲率进行估计, 然后根据最大和最小主曲率求出 此点的 SI, 并通过相关面和反向点计算出此点的 SDF. 其次, 通过谱聚类将点云模型过分割为模型 子块, 用 GS, SI 和 SDF 构造特征张量表示模型的 每个子块, 并用张量范数及其分量范数得到一组 相似的块. 最后, 利用张量和 $L_{1}$ 中值构造一个融 合特征形状描述符，并由此得到模型子块的相似 性. 以牛模型为例, 主要过程如图 1 所示, 其中, 图 1d 所示为通过谱聚类对模型进行过分割成模型 子块(100 块); 图 1e 所示三阶特征张量由 GS, SI 和 $\mathrm{SDF}$ 组成; 图 $1 \mathrm{f}$ 所示为通过局部形状描述符实现 自相似性分析，颜色越接近，相似性越高; 图 $1 \mathrm{~g}$ 所示为以右角(红色)为参考块进行相似块匹配, 其 中得到左角(蓝色)是最相似的块.

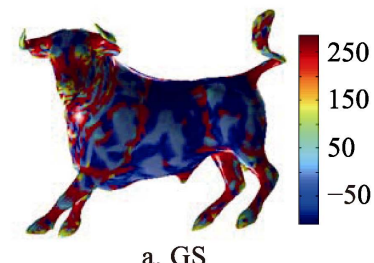

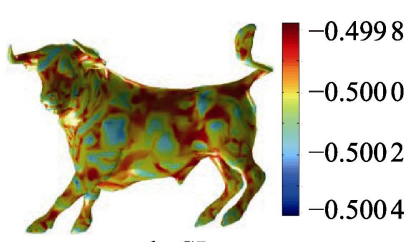

b. SI

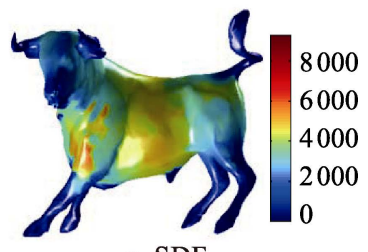

c. SDF

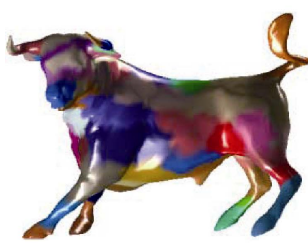

d. 点云模型过分割

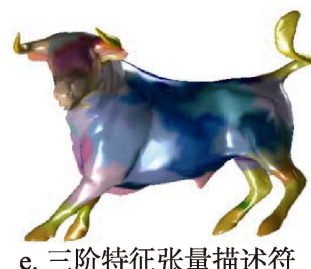

量描述符

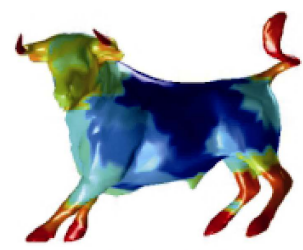

f. 自相似性分析

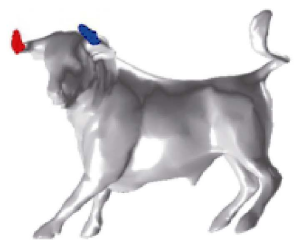

g. 相似块匹配

图 1 本文方法构造流程

\section{2 点云模型曲率估算}

在数字几何处理领域，曲率是模型曲面形状 的内蕴几何量, 能反映出模型表面的凸凹特点. 本 文对 Meyer 等 ${ }^{[20]}$ 给出三角网格模型的近似计算方 法加以改进, 得到点云模型 GS 的计算方法. 首先 对于顶点 $x$ 使用 $K$ 近邻 $(K$-nearest neighbor, $K N N)$ 算法找其 $K$ 近邻点, 进行 Delaunay 三角剖分，再利 用文献[20]中的公式得到 $\mathrm{GS}$ 和平均曲率 $k_{\mathrm{G}}$ 和 $k_{\mathrm{M}}$, 用曲率值进行着色，如图 $2 \mathrm{a}$ 和图 $2 \mathrm{~b}$ 所示. 进而可 得最大-最小主曲率分别为 $k_{1}=k_{\mathrm{M}}+\sqrt{k_{\mathrm{M}}^{2}-k_{\mathrm{G}}}$, $k_{2}=k_{\mathrm{M}}-\sqrt{k_{\mathrm{M}}^{2}-k_{\mathrm{G}}}$.

\section{3 点云模型 SI 的计算}

除了经典的表面曲率测度(如 GS 和平均曲率) 能很好地表征局部形状外，仅用最大-最小主曲率 构建的 SI 也可作为描述三维模型表面凹凸程度的 参数 ${ }^{[21]}$, 能客观地反映三维模型表面任意曲面的 形状. 利用第 2.2 节的最大-最小主曲率 $k_{1}, k_{2}$, 得 到点云模型 SI 的计算式为

$$
\mathrm{SI}= \begin{cases}-\frac{2}{\pi} \arctan \frac{k_{1}+k_{2}}{k_{1}-k_{2}}, & k_{1} \neq k_{2} \\ 0, & k_{1}=k_{2}=0 \\ -1, & k_{1}=k_{2}>0 \\ 1, & k_{1}=k_{2}<0\end{cases}
$$




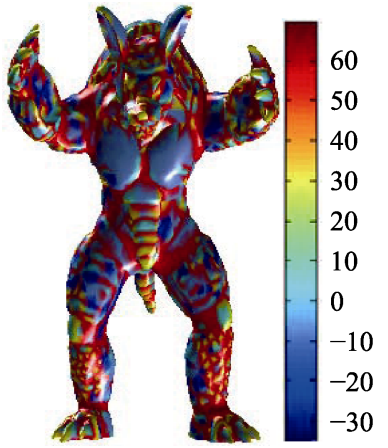

a. GS

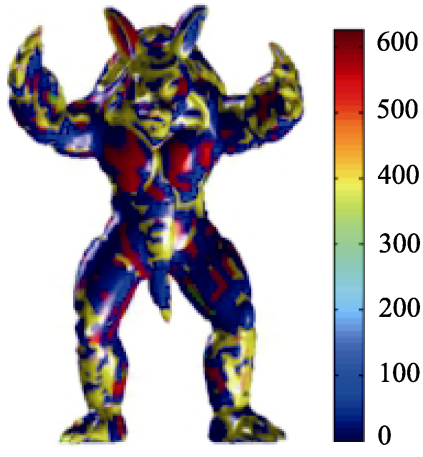

b. 平均曲率

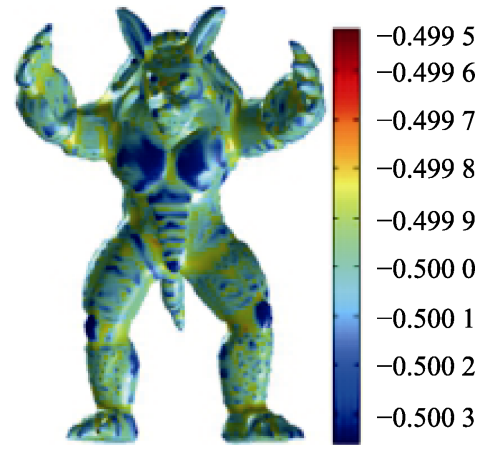

c. SI

图 $2 \mathrm{GS}$ 、平均曲率和 SI 图谱

SI 的取值范围为 $-1 \sim 1$, 取值越接近 1 , 说明 点云模型表面点越凸; 反之, SI 的取值越接近 -1 , 则表示模型表面越凹; 若 $k_{1}=k_{2}=0$, 表明表面没 有弯曲, 是平面点, 此时令 SI $=0$. SI 图谱如图 $2 \mathrm{c}$ 所示.

\section{4 点云模型 SDF 的计算}

SDF 也是形状描述的重要几何特征 ${ }^{[9,22]}$, 它对 物体姿态的变化是不变的, 可以同时描述三维模 型的内部区域和边界部分. 因此, 它在计算机图形 学领域有着广泛的应用. Shapira 等 ${ }^{[9]}$ 给出了在网格 模型上计算 SDF 的方法, 对于网格模型中的每个 点, 以此点为顶点的圆雉体中多条射线的长度之 和定义为该顶点的 SDF 值. 但是, 射线与网格模 型之间的交点不能应用于三维点云模型. 本文提 出一种利用相关面和反向点计算点云模型 SDF 的 方法.

首先根据模型的轴向对齐最小包围盒 (axis-aligned bounding box, AABB) 定义相关面，该 包围盒是一个包围物体的长方体, 3 个轴向量分别 与世界坐标系的轴平行. 对于 $\mathrm{AABB}$ 的面 $S_{j}$, 假 设其法向量为 $N_{S_{j}}$; 对于点 $x$, 其法向量为 $N(x)$. 如果 2 个法向量之间的夹角小于 $\pi / 2$, 定义平面 $S_{j}$ 是相对于点 $x$ 的一个相关面(relevant facet, RF). 显 然, 对于给定的包围盒中的一个点, 最多有 3 个 RF. 图 3 所示为一个点有 $1 \sim 3$ 个 RF 的 3 种情况, 其 中, 红色点和箭头线是特征点及其法向量, 蓝色箭 头线是 $\mathrm{RF}$ 的法向量.

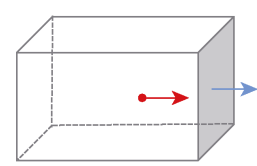

a. 1 个相关面 b. 2 个相关面

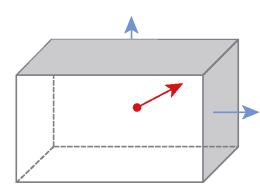

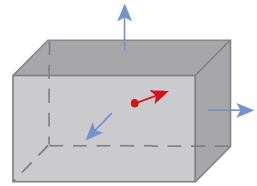

c. 3 个相关面
图 33 种情况下的相关面
然后给出了反向点的定义. 对于点 $x$, 如果点 $P$ 的法向与点 $x$ 的法向相反(即 2 点法向量之间的 夹角在 $(\pi / 2, \pi)$ ), 且连接 $P$ 和 $x$ 的线段上的点都在 模型内, 则 $P$ 称为点 $x$ 的反向点, 记作 $P_{\text {ant }}(x)$. 图 4 给出了一个点及其反向点的例子, 这里从包围盒 的俯视图中显示了点 $x$ 和 RF. 可以发现, 点 $P_{1}$ 是 点 $x$ 的反向点, 但只根据法向量方向判断反向点, 则会出现一些伪反向点 (如图 4 中的点 $P_{2}$ 和 $P_{3}$ ). 对 于点云模型，可以使用 $\mathrm{RF}$ 来消除伪反向点，即

$$
P_{\text {ant }}=\left\{P \mid \cos \angle(N(P), N(x))<0, D\left(P, \mathrm{RF}_{j}\right)>\right.
$$$$
\left.D\left(x, \mathrm{RF}_{j}\right), j=1,2,3, P \in \text { point cloud }\right\}
$$

其中, $D(\cdot)$ 为欧几里得度量运算符; $\mathrm{RF}_{j}$ 是点 $x$ 的第 $j$ 个相关面. 如果点 $P$ 满足条件 $\cos \angle(N(P)$, $N(x))<0$, 即对应点 $P$ 和 $x$ 的 2 个法向量之间的 夹角在 $(\pi / 2, \pi)$ 之间, 并且点 $P$ 和 $R F$ 之间的距离 大于点 $x$ 和 RF 之间的距离，即

$$
D\left(P, \mathrm{RF}_{j}\right)>D\left(x, \mathrm{RF}_{j}\right), j=1,2,3 .
$$

则此点被定义为反向点 $P_{\mathrm{ant}}$.

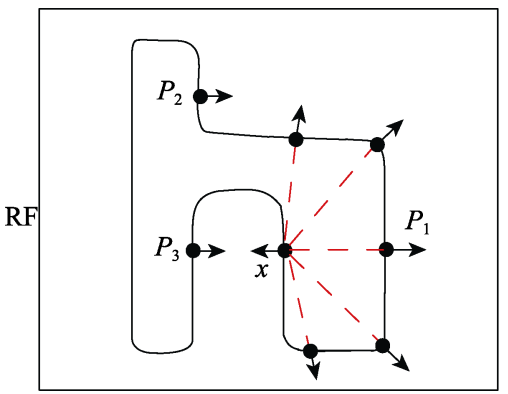

图 4 利用 $\mathrm{RF}$ 和反向点计算 $\mathrm{SDF}$

从点 $x$ 到集合 $P_{\text {ant }}(x)$ 的所有距离都按升序计 算和排列. 只有距离小于 $\alpha d_{\text {min }}$ 的点被选作 SDF 计 算的贡献点, 其中 $\alpha$ 是一个國值, 通常设置为 $\alpha=5, d_{\min }$ 是点 $x$ 到 $P_{\mathrm{ant}}(x)$ 中点的最小距离. 例 
如, 在图 4 中, 点 $x$ 和 $P_{1}$ 之间的距离是由点 $x$ 和集 合 $P_{\text {ant }}(x)$ 中的点组成的所有线段的最小距离. 对 于大多数点云模型，给定点的反向点数小于 30 . 如果反向点的数目大于 30 , 则根据长度选择前 30 个点作为贡献点, 这足以进行 SDF 计算. 这样可 以在不影响局部形状分析的情况下，尽可能地提 高计算效率.

点 $x$ 的 SDF 值是通过求出贡献点与点 $x$ 之间 的距离之和得到的, 因此 SDF 值可以反映三维模
型表面的局部厚度, 并且可以将其可视化为彩色 图像, 如图 5 所示. 可以看出, Shapira 等 ${ }^{[9]}$ 和张猛 等 ${ }^{[22]}$ 的研究结果中, 兔子面部和背部有相似的 SDF 颜色，这与这些区域厚度不同的形状特征不 一致. 本文的结果更加合理, 可以在模型的面部和 背部不同地表达形状. 此外, 本文方法避免了寻找 圆雉内射线与模型表面交点的步骤，因此与文献 [9,22]中的 SDF 计算方法相比，本文的 SDF 计算是 方便和准确的.

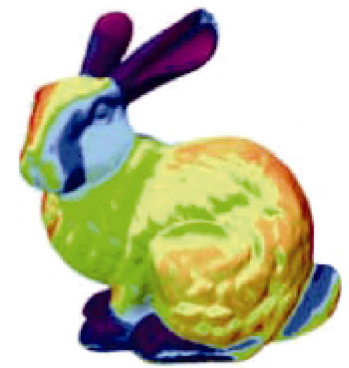

a. 文献[9]

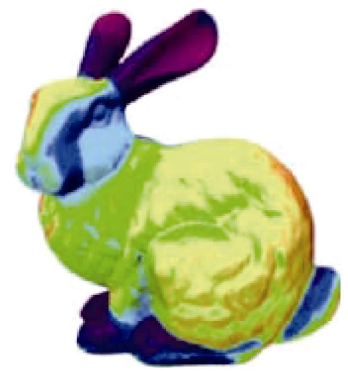

b. 文献[22]

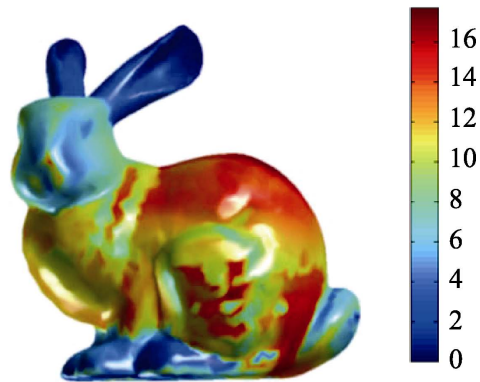

c. 本文

图 5 不同 SDF 计算方法的比较

\section{5 点云模型过分割}

假设 $R$ 代表一个点云模型． $R$ 的过分割可以 看做将 $R$ 划分为 $n$ 个非空子集 $R_{1}, R_{2}, \cdots, R_{N}$, 满 足以下条件.

(1) $\bigcup_{i=1}^{N} R_{i}=R$;

(2) $\forall i, j, i \neq j$, 有 $R_{i} \cap R_{j}=\varnothing$;

(3) 对于 $i=1,2, \cdots, N, P\left(R_{i}\right)=$ TRUE, 其中, $P\left(R_{i}\right)$ 是集合 $R$ 中元素的逻辑谓词.

条件(1)表明分割的所有子区域的并集是原始 模型，使分割过程中的每个点都得到处理; 条件 (2)表示分割结果中的每个区域不重叠; 条件(3)表 示分割结果中的每个区域 $\left(R_{i}\right)$ 具有相同的属性, 可以聚为一类. 因此, 点云模型的过分割可以表示 为子空间聚类和精确覆盖问题 ${ }^{[18]}$. 过分割可以将 物体分割成更为精细的单元，这对于三维模型的 自相似性分析非常有用.

现有一些经典算法，如 $K$-means 法、层次聚类 法、DBSCAN 方法等实现了数据聚类. 与这些方法 相比, 谱聚类具有更好的性能(如在较低维度上进 行聚类, 并保留模型的局部特征和拓扑结构)并已 应用于多个领域中 ${ }^{[23]}$. 本文利用文献[24]的思想, 使用谱聚类分割点云模型. 需要注意的是, 它需要 谱聚类中的图形具有连通性. 对于点云模型中的 一个点，根据该点周围的 $K \mathrm{NN}$ 构造点的图连通性. 在谱聚类过程中，选取前 7 个特征值，将模型划分
为 $N$ 个子块(本文设为 100 个子块). 图 6 显示了过 分割的结果，其中不同的块由不同的颜色表示.

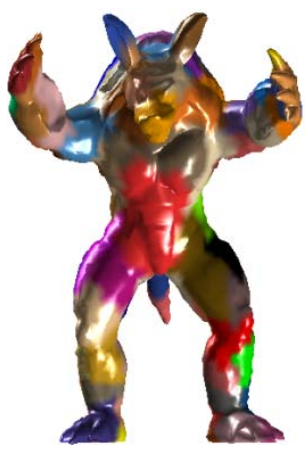

图 6 基于谱聚类的点云模型过分割结果

值得注意的是，聚类生成子块的质量会影响 局部描述符的质量, 进而影响自相似性分析结果. 图 7 所示为本文对不同聚类方法的对比, 如图 7a 所示左右胸部、两膝盖相对应的分割块颜色差异较 大，说明不匹配; 类似的情况在图 $7 \mathrm{~b}$ 所示 2 只耳 朵、图 7c 所示膝盖和胸部处也有不匹配的情况出 现; 经比较, 图 $7 \mathrm{~d}$ 所示分割块是最好的, 能得到 较好的匹配.

\section{6 点云模型的特征张量构造}

张量理论是数学的一个重要分支, 广泛应用 于模式识别、图像去噪、数据降维等多方面 ${ }^{[25]}$. 本 文利用张量表示三维物体的几何特征, 并进行相 似性分析。 


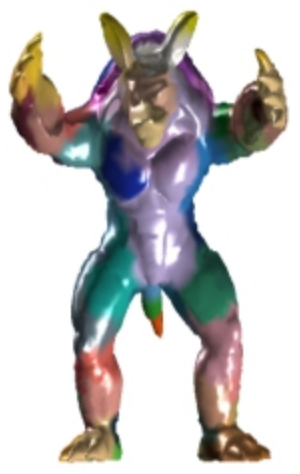

a. DBSCAN 聚类

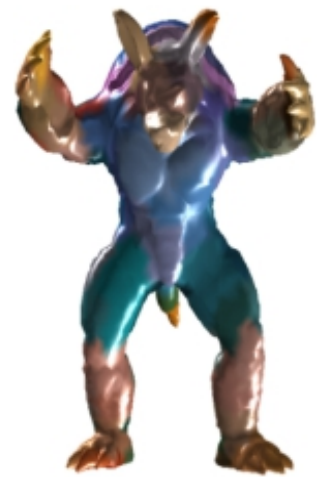

b. 层次聚类

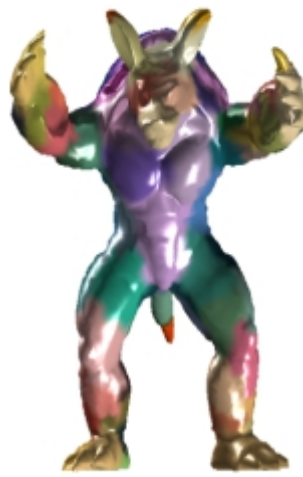

c. $K$-means 聚类

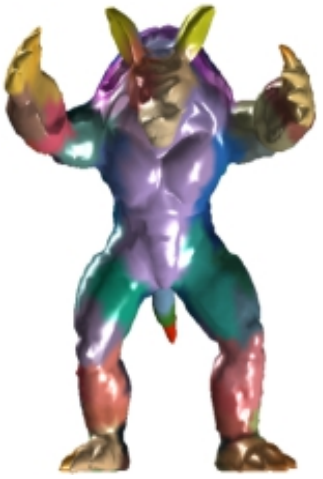

d. 本文

图 7 文献[23]中不同聚类方法与本文方法对模型过分割结果的影响比较

根据上述计算点云模型上各点几何特征 $(\mathrm{GS}$, SI 和 SDF)的方法, 构造张量表示模型的各子块. 三维模型中每个点的局部形状描述符与其相邻区 域有关，因此对于给定点的 GS, SI 和 SDF，使用其 $K \mathrm{NN}$ 和子块构成此点的几何性质，然后构造三阶 张量进行形状分析. 步骤如下: 首先用 $K \mathrm{NN}$ 算法 得到各点的 $K$-近邻，然后根据谱聚类将点云模型 聚类，划分为 $N$ 个模型子块. 在每个模型子块 $i(i=1,2, \cdots, m)$ 中, $m$ 是子块 $i$ 中的点数; 每个点 $P$ 及其 $K$-近邻的 GS 形成一个列向量，所有列向量 形成一个 GS 矩阵

$$
\mathbf{G S}_{i}=\left(\begin{array}{cccc}
G_{01} & G_{02} & \cdots & G_{0 m} \\
G_{11} & G_{12} & \cdots & G_{1 m} \\
\vdots & \vdots & \cdots & \vdots \\
G_{k 1} & G_{k 2} & \cdots & G_{k m}
\end{array}\right) .
$$

其中, $G_{0 t}$ 是模型子块 $i$ 内的第 $t$ 个点的 $\mathrm{GS} ; G_{s t}$ 是 第 $t$ 个点的第 $s$ 个近邻点的 GS, $s=1,2, \cdots, k$, $t=1,2, \cdots, m, m$ 是模型子块 $i$ 内的点数. 对于 $\mathbf{S I}_{i}$ 和 SDF $_{i}$ 有类似的构造方法，如

$$
\begin{aligned}
\mathbf{S I}_{i} & =\left(\begin{array}{cccc}
\mathrm{SI}_{01} & \mathrm{SI}_{02} & \cdots & \mathrm{SI}_{0 m} \\
\mathrm{SI}_{11} & \mathrm{SI}_{12} & \cdots & \mathrm{SI}_{1 m} \\
\vdots & \vdots & \cdots & \vdots \\
\mathrm{SI}_{k 1} & \mathrm{SI}_{k 2} & \cdots & \mathrm{SI}_{k m}
\end{array}\right), \\
\mathbf{S D F}_{i} & =\left(\begin{array}{ccccc}
\mathrm{SDF}_{01} & \mathrm{SDF}_{02} & \cdots & \mathrm{SDF}_{0 m} \\
\mathrm{SDF}_{11} & \mathrm{SDF}_{12} & \cdots & \mathrm{SDF}_{1 m} \\
\vdots & \vdots & \cdots & \vdots \\
\mathrm{SDF}_{k 1} & \mathrm{SDF}_{k 2} & \cdots & \mathrm{SDF}_{k m}
\end{array}\right) .
\end{aligned}
$$

对于分割块 $i$, 由 $\mathbf{G S}_{i}, \mathbf{S I}_{i}, \mathbf{S D F}_{i}$ 作为页 (page)构成 $(k+1) \times m \times 3$ 的三阶张量 $\boldsymbol{T}^{(i)}$, 即

$$
\begin{aligned}
& \boldsymbol{T}^{(i)}(:,:, 1)=\mathbf{G S}_{i}, \\
& \boldsymbol{T}^{(i)}(:,:, 2)=\mathbf{S I}_{i}, \\
& \boldsymbol{T}^{(i)}(:,:, 3)=\mathbf{S D F}_{i} .
\end{aligned}
$$

其中, $i=1,2, \cdots, N$.

\section{7 基于张量的点云模型相似块匹配}

对于张量 $\boldsymbol{X}=(X(i, j, h))_{(k+1) \times m \times 3}$, 其 Frobenius 范数 ${ }^{[23]}$ 为 $\|\boldsymbol{X}\|=\sqrt{\sum_{h=1}^{3} \sum_{i=1}^{k+1} \sum_{j=1}^{m} X(i, j, h)^{2}}$.

利用张量范数公式求出每个模型子块的张量 范数, 再由张量范数距离得到初始相似块 (20 个子 块), 记为 $S_{\text {pre }}$. 再分别求出 GS 矩阵、SI 矩阵、SDF 矩阵(即张量的 1, 2, 3 页)的范数, 分别记为 $n_{1}, n_{2}$, $n_{3}$, 只要

$$
\frac{\max _{v \in S_{\mathrm{pre}}(u)}\left(n_{i}(u), n_{i}(v)\right)}{\min _{v \in S_{\mathrm{pre}}(u)}\left(n_{i}(u), n_{i}(v)\right)}>\beta_{i}, i=1,2,3
$$

其中, $u$ 为参考块; $S_{\mathrm{pre}}(u)$ 为 $u$ 的初始相似块集 合; $\beta_{i}(i=1,2,3)$ 为阈值，一般分别取为 $28,4,8$. 表明 $u, v$ 在 $n_{i}$ 方面的相似性较差, 就将 $v$ 从 $S_{\text {pre }}(u)$ 中剔除掉. 由于 $\mathrm{GS}$ 和 SI 仅能反映模型局部特性, 而 SDF 能反映模型总体特性, 因此再利用与参考 块 $u$ 的 SDF 矩阵范数 $n_{3}$ 距离从小到大排序, 得到 相似程度从强到弱排列的精确相似块集合.

利用此方法在一些 $3 \mathrm{D}$ 点云模型上实验，得到 如图 8 所示比较好的效果, 其中, 红色为参考块, 其他颜色为参考块的相似块. 可以看出, 该方法对 相似子块具有较精确的匹配.

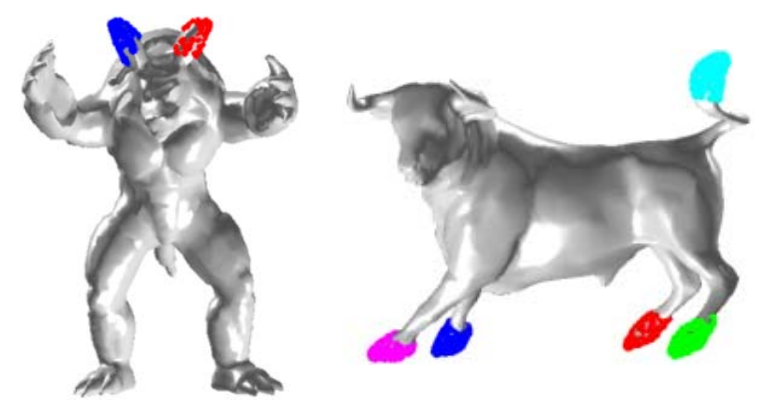

图 8 参考块及其相似块匹配 


\section{8 融合特征形状描述符和自相似性分析}

单特征描述符 GS, SI 或 SDF 不能很好地区分 子块的相似性，上述方法使用张量范数及其分量 范数搜索给定参考块的相似块集. 本节使用张量 和 $L_{1}$ 中值构造局部形状描述符，它不仅可以确定 $3 \mathrm{D}$ 模型的相似部分，而且可以可视化 3D 模型并反 映对象的局部形状分布. 为了定量描述模型的局部 形状, 将特征张量的分量范数 $n_{i}(i=1,2,3)$ 与 $L_{1}$ 中 值相结合, 构造了模型任意子块的融合特征描述符.

这里参考 SI 公式(1)，并受文献[26]启发，使用 $L_{1}$ 中值并结合张量定义形状描述符. $L_{1}$ 中值是统计 学中常见的统计变量, 是给定点集的唯一的全局 中心，能反映一组数据的集中趋势，因此不易受到 数据中极值的影响 ${ }^{[27]}$. 在这里, 构造

$$
\begin{aligned}
D_{i}^{(u)} & =\frac{2}{\pi} \arctan \frac{n_{i}}{M\left(\left\{n_{i}(u) \mid u=1,2, \cdots, N\right\}\right)}, \\
u & =1,2, \cdots, N, i=1,2,3
\end{aligned}
$$

其中, $M(x)$ 为数据集 $x$ 的 $L_{1}$ 中值, $N$ 为子块的 数量. 根据式 $(4)$, 将 $n_{i}$ 映射为 $(0,1)$ 中的数, $D^{(u)}=$ $\left(D_{1}^{(u)}, D_{2}^{(u)}, D_{3}^{(u)}\right)$ 为模型子块 $u$ 的形状描述符, 并由 其分别作为颜色分量 RGB 值可视化模型. 从图 9 可以看出，此描述符可以很好地表征模型的各个 部分，对于部位相同的子块，其颜色也相近；对于 部位相差较大的子块，其颜色也相差较大

为了表示模型子块之间的相似性程度，定义 相似性测度为

$$
M_{\text {sim }}=1-\boldsymbol{D} . / \max (\boldsymbol{D})
$$

其中, ./ 为点除运算, 即矩阵 $\boldsymbol{D}$ 中每个元素都要 除以某个数; $\boldsymbol{D}$ 为模型不同子块描述符的欧几里 得度量矩阵; $\max (\boldsymbol{D})$ 是所有子块间距离的最大值; D. $/ \max (\boldsymbol{D})$ 是将 $\boldsymbol{D}$ 进行归一化, 即矩阵 $\boldsymbol{D}$ 中每个 元素都要除以 $\boldsymbol{D}$ 中的最大值. $M_{\text {sim }}$ 越接近 1,2 个 子块越相似； $M_{\mathrm{sim}}$ 越接近 0,2 个子块相似性越差. 图 10 所示为模型子块之间的相似性度量, 其中蓝 色箭头指向参照块; 颜色越接近, 子块越相似.
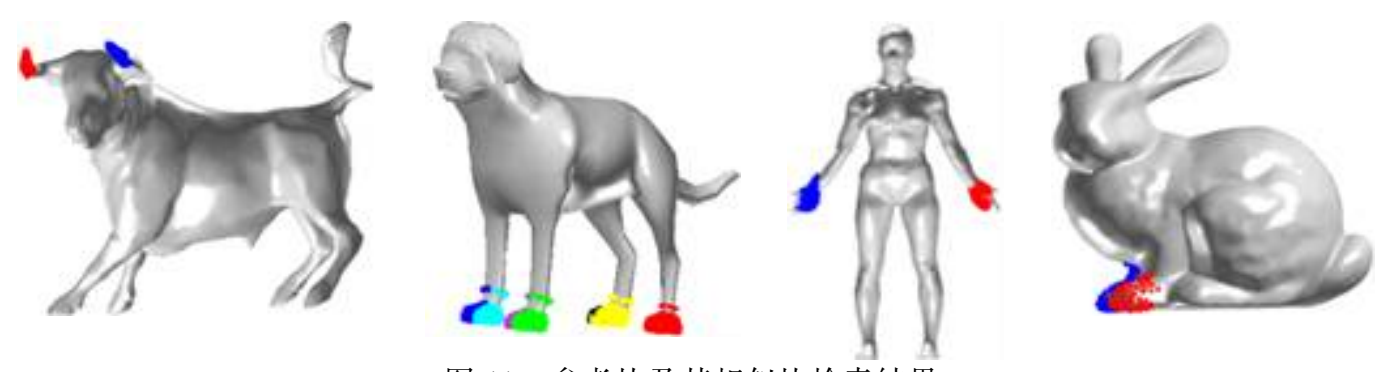

图 11 参考块及其相似块检索结果

\section{3 结果与讨论} $8 \mathrm{~GB}$ 内存的计算机上实现本文方法，在各种 $3 \mathrm{D}$ 模 型上进行测试, 这些 3D 模型是从普林斯顿大学形 状基准库 ${ }^{\circledR}$ 中获得的. 将得到的结果与一些常用的 自相似性分析方法进行比较，包括基于局部形状 描述子的部分匹配 ${ }^{[3,5-6]}$ 和基于局部形状描述子的 显著性检测 ${ }^{[10-12]}$. 需要注意的是, 本文的局部自相 似分析方法直接应用于点云模型，这意味着它不 会将点云模型转换为网格模型, 为了渲染效果好, 本文只是使用了它们的网格表示进行更直观的可 视化.

首先, 利用特征张量描述符的第 $1,2,3$ 分量范 数方法得到模型子块的精确相似块. 图 11 表明, 前 3 个模型中具有明显位置差异的相似区域可以 得到精确匹配, 第 4 个模型中具有粘连的前 2 条腿 也可以精确匹配; 然后，使用这个构造的形状描述 符来表征 3D 模型. 如图 12 所示, 每个区域的局部 形状描述符可以用式(4)中的向量表示; 用 RGB 颜 色显示, 颜色越接近, 子块越相似. 最后, 使用式 (5)实现自相似性分析, 结果如图 13 所示, 其中蓝 色箭头所指的是参考块. 对于更多的点云模型, 在 图 14 中给出了形状描述符和自相似性分析的结果. 由此可见, 本文方法可以得到满意的自相似性分 析结果.

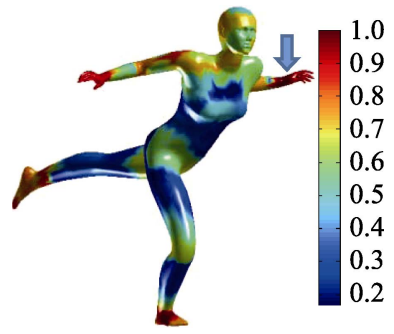

图 10 子块间的相似性度量

在 Intel Core i7-4710mq CPU@2.50 GHz 和

(1) http://Shape.cs.Princeton.edu/Benchmark/ 


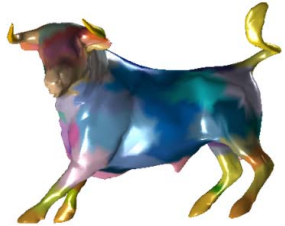

图 12 形状描述符可视化示例

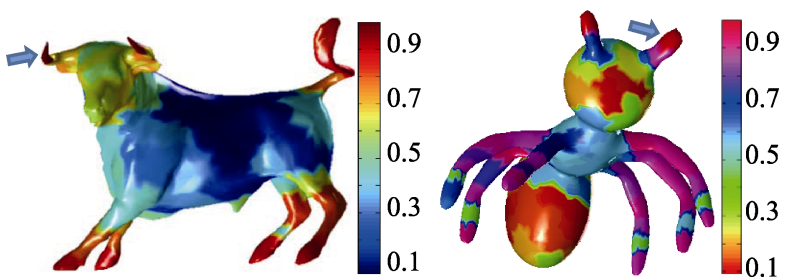

图 13 子块之间的相似性匹配
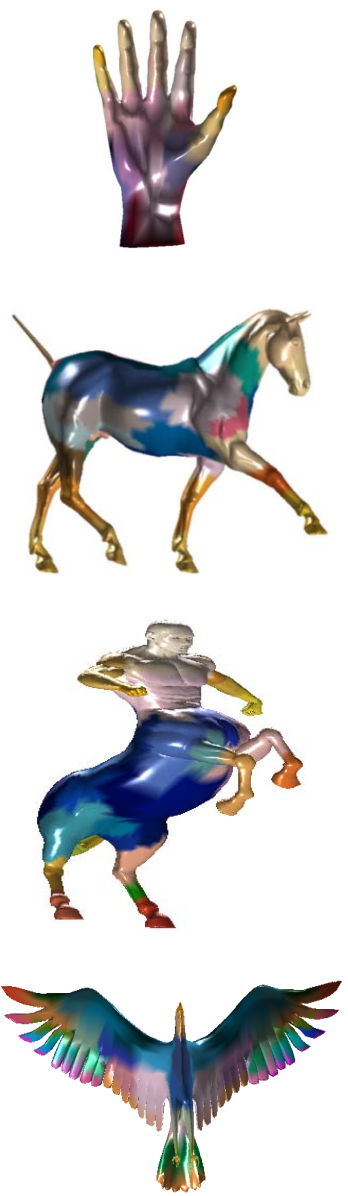

a. 本文形状描述符可视化

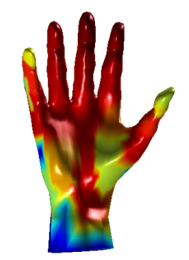

中指头为参考块
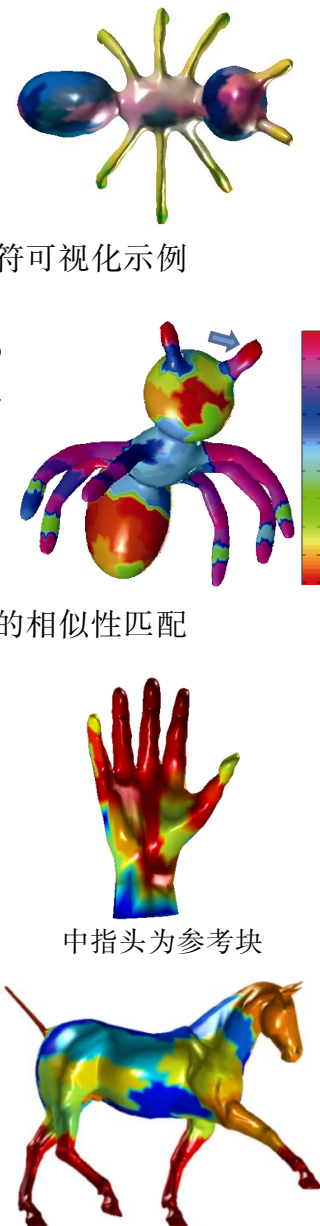

左前足为参考块
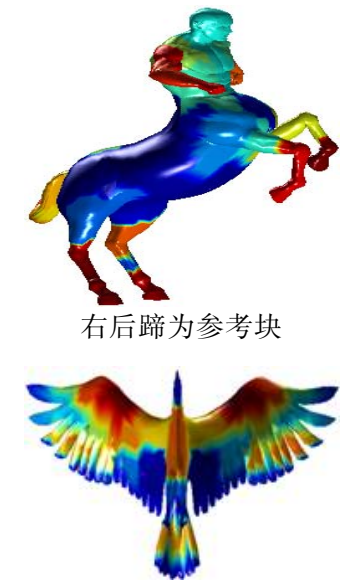
色最深处

b. 参考块和其他区域的 自相似性分析结果
参考块是左翼的上边缘, 颜

图 14 3D 点云模型自相似性的更多结果

\section{1 与其他描述符的比较}

为了验证形状描述符的有效性, 首先与其他 单个特征描述符进行了比较, 分别用 GS 矩阵、SI 矩阵、SDF 矩阵和张量的范数进行比较. 对于图 15 所示的犰狳模型, 可以发现模型的头部和耳朵
用 GS 矩阵范数不能区分出来. 根据 SI 矩阵范数, 发现头部与腿部相似，嘴巴与耳朵和脚趾相似，这 与形状特征不一致. 根据 SDF 矩阵范数, 发现左 脚与右腿下部相似, 这也是不合理的. 根据张量范 数, 发现腹部与脚趾和左手相似, 这是错误的匹 配. 在图 $15 \mathrm{e}$ 中, 发现由特征张量的 3 个分量范数 及 $L_{1}$ 中值变换的描述符可以有效地表示模型的局 部自相似性. 值得注意的是, 图 15a 图 15d 中的形 状描述符由一个单一特征计算, 每个子块由一个值 表示，并根据所有子块的值和范围由颜色设置. 图 $15 \mathrm{e}$ 中的形状描述符由向量计算，每个子块由 RGB 颜色设置. 此外, 还对不同层的特征张量的形状表 达能力进行了分析对比. 图 16a 图 16c 是仅由 1 层 张量产生的结果; 图 16d 图 16f 是由 2 层张量产生 的结果; 图 $16 \mathrm{~g}$ 是由 3 层张量产生的结果. 可以发 现，由 3 层构成的张量描述符具有较好的形状表达 分析能力，能够区分物体的不同部分.

\section{2 与其他方法比较}

为了验证本文方法的优越性，与现有的自相 似性分析方法进行了比较. 与图 17 图 19 中其他形 状描述符方法相比, 可以发现本文方法能够更好地 描述不同的子块，更准确地匹配参考块的相似块.

首先分析了狗模型的自相似性，并与文献[11] 方法进行了比较. 如图 17 所示, 文献[11]方法无法 在模型中准确地找到类似的子区域. 例如, 以左后 腿为参考块，后腿与前腿不相似，但与鼻子、嘴和 耳朵相似. 相比较而言, 本文方法可以发现前腿与 后腿相似。

针对图 18 所示的佛像模型, 将本文方法与文 献 $[3,6]$ 的部分匹配方法进行了比较. 当将一个花 部分标记为参考块时, 可以检测到模型中其他 3 个 花部分，这与其他 2 种方法相同. 本文方法的优点 是可以同时处理点云模型和网格模型，而文献 $[3,6]$ 中的方法仅适用于网格模型.

然后选择犰狳模型作为案例, 将本文方法和 文献[5-6,10-12]中的方法进行比较. 把模型的右手 作为参照块, 其他 5 种方法都不能得到精确的相似 性匹配，甚至得到错误的匹配. 例如，文献[5]方法 结果中, 右手不是与左手最相似, 而与左脚最相 似; 文献[6]方法结果中, 1 只耳朵和 2 只脚与右手 相似; 文献[10]方法结果中的嘴和脸与右手相似; 文献[11]方法结果中的右手和 2 只脚更相似, 而不 是与左手更相似; 文献[12]中的嘴和手相似. 相比 之下，本文方法可以得到与右手最相似的部分是 左手, 如图 19 所示. 


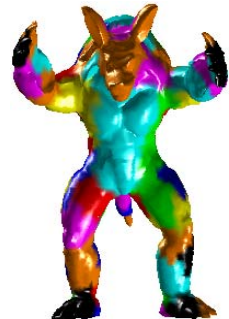

a. GS 矩阵范数

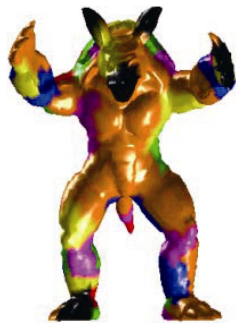

b. SI 矩阵范数

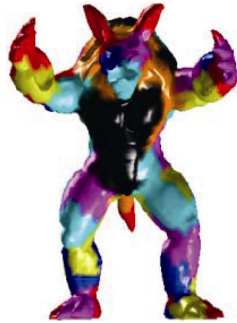

c. SDF 矩阵范数

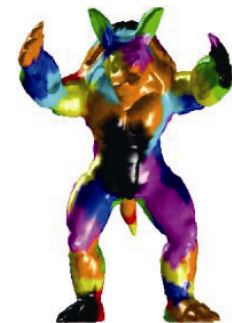

d. 张量范数

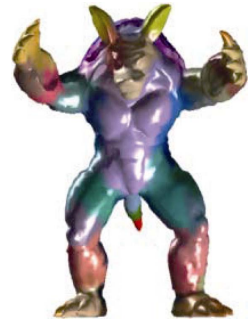

e. 局部形状描述符

图 15 几种特征描述符的比较

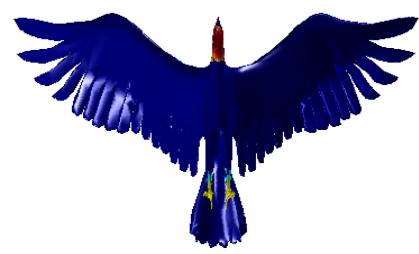

a. 1 层(GS)张量

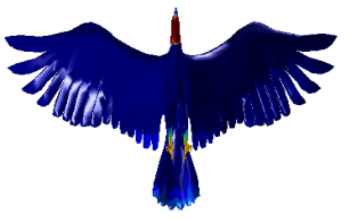

d. 2 层 $(\mathrm{GS}+\mathrm{SI})$ 张量

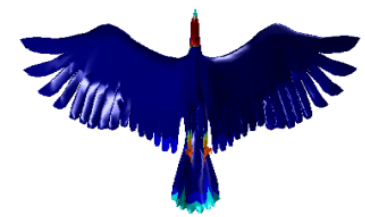

e. 2 层 $(\mathrm{GS}+\mathrm{SDF})$ 张量

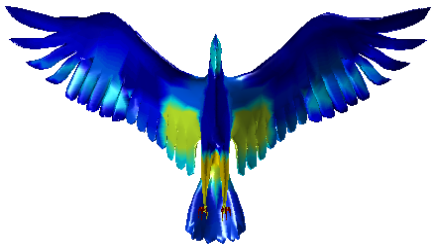

b. 1 层(SI)张量

图 16 不同层的特征张量的形状表达分析能力对比

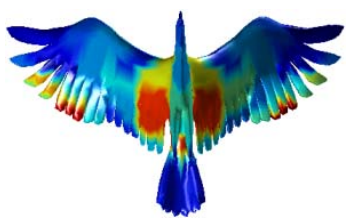

f. 2 层 $(\mathrm{SI}+\mathrm{SDF})$ 张量

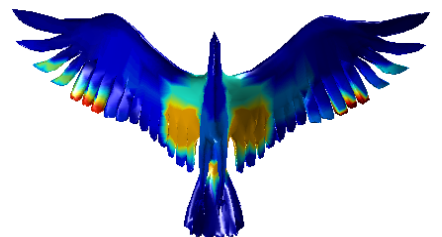

c. 1 层(SDF)张量

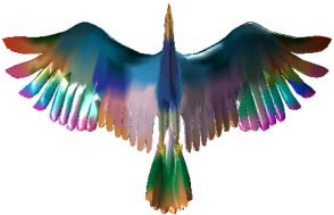

g. 3 层 $(\mathrm{GS}+\mathrm{SI}+\mathrm{SDF})$ 张量

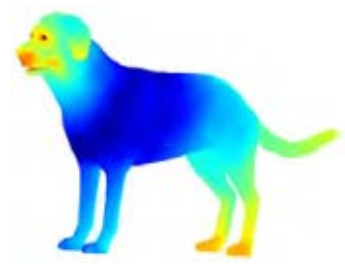

a. 文献[11]

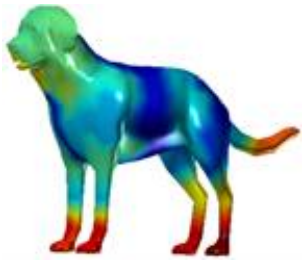

b. 本文
图 17 本文与文献[11]方法的自相似性结果比较

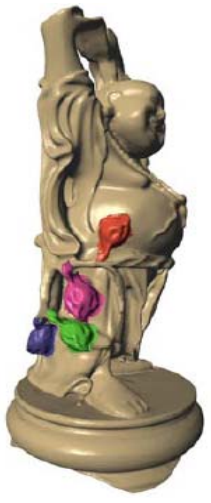

a. 文献 [6]中的 部分匹配

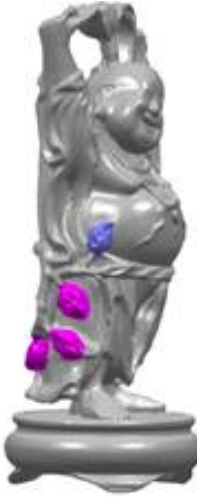

b. 文献[3]中的 部分匹配

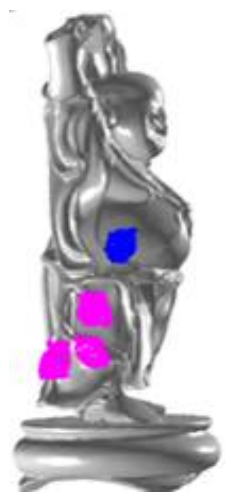

c. 本文
图 18 本文方法与其他部分匹配方法的匹配结果比较

除了进行定性比较和分析外, 还对图 19 的犰 狳模型相似性分析的几种方法进行了定量比较和 分析, 如表 1 所示. 这里用点的相对误差 $E_{\mathrm{p}}$ 和面
积的相对误差 $E_{\mathrm{a}}$ 进行评价, 它们分别定义为

$$
\begin{gathered}
E_{\mathrm{p}}=\left|N_{\mathrm{s}}-N_{\mathrm{r}}\right| / N_{\mathrm{r}} \\
E_{\mathrm{a}}=\left|A_{\mathrm{s}}-A_{\mathrm{r}}\right| / A_{\mathrm{r}}
\end{gathered}
$$

其中, $N_{\mathrm{s}}$ 和 $N_{\mathrm{r}}$ 分别为相似块和参考块中点的个 数; $A_{\mathrm{s}}$ 和 $A_{\mathrm{r}}$ 分别为相似块和参考块的面积.

对于图 19 所示的犰狳模型，将右手设置为参 考块，参考块中的点数和面积如表 1 第 2 列和第 3 列所示. 表 1 第 4 列至第 7 列显示了所有相似块中 的点数、所有相似块中的面积、点的相对误差和面 积的相对误差. 可以发现本文方法中, 所有相似块 中点的数目最接近于参考块中点的数目, 所有相 似块的面积最接近于参考块的面积. 因此, 本文方 法的 $E_{\mathrm{p}}$ 和 $E_{\mathrm{a}}$ 最小, 这意味着它能够得到令人满意 的自相似性分析结果.

最后，对本文方法进行复杂度分析并与其他 自相似性分析方法进行运行效率比较. 虽然本文 方法通过张量形式将 3 个特征进行整合增加了步 骤，却减少了对 SDF 特征的计算，因此时间复杂 度为 $O(\log n)$; 其中, $n$ 为模型过分割子块个数. 本文的自相似性分析方法的运行时间与现有的部 分匹配和显著性检测方法没有太大的不同，如表 2 所示. 其中, 3 个模型的点数分别为 5000,1618 , 20000 ; 面片数分别为 $9996,3220,40412$. 


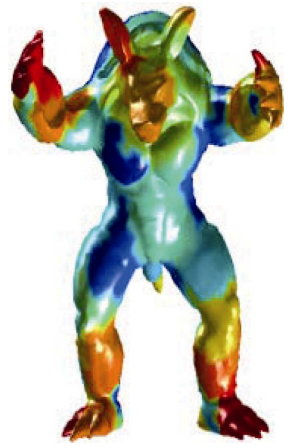

a. 文献 $[5]$

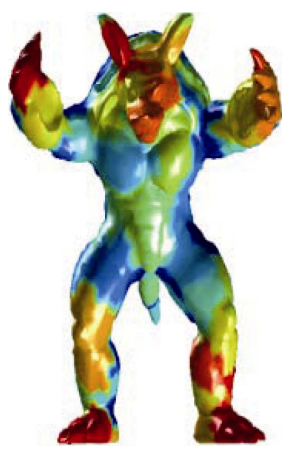

d. 文献 $[11]$

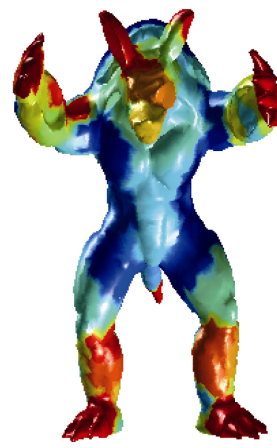

b. 文献 $[6]$

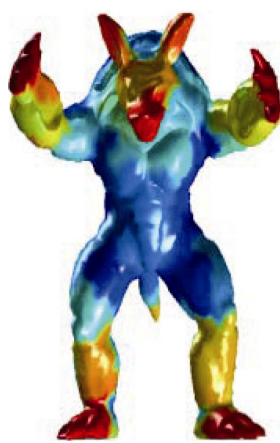

e. 文献 $[12]$

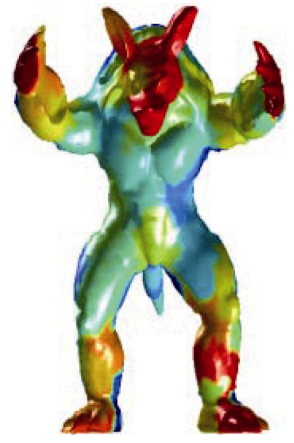

c. 文献[10]

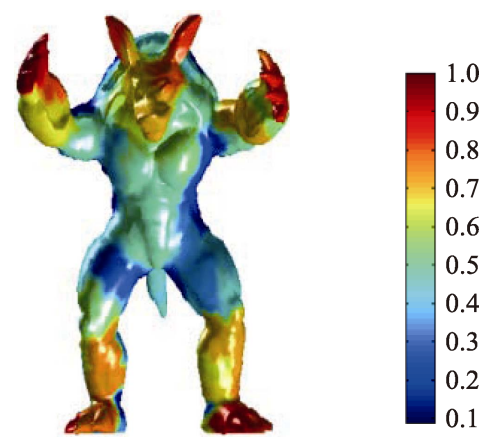

f. 本文

图 196 种自相似性分析方法的比较

表 16 种不同方法对犰狳模型的定量分析比较

\begin{tabular}{lcccccc}
\hline \multicolumn{1}{c}{ 方法 } & $N_{\mathrm{r}}$ & $A_{\mathrm{r}}$ & $N_{\mathrm{s}}$ & $A_{\mathrm{s}}$ & $E_{\mathrm{p}}$ & $E_{\mathrm{a}}$ \\
\hline 文献[5] & 153 & 10727.3 & 115 & 15070.3 & 0.248366 & 0.404855 \\
文献[6] & 164 & 10169.1 & 317 & 23546.8 & 0.932927 & 1.315524 \\
文献[10] & 183 & 13966.9 & 201 & 13999.3 & 0.098361 & 0.002320 \\
文献[11] & 221 & 16240.2 & 480 & 19393.3 & 1.171946 & 0.194154 \\
文献[12] & 182 & 13063.7 & 404 & 30292.4 & 1.219780 & 1.318822 \\
本文 & 158 & 11299.7 & 165 & 11275.6 & 0.044304 & 0.002133 \\
\hline
\end{tabular}

表 26 种不同方法运行时间比较

\begin{tabular}{lccc}
\hline \multirow{2}{*}{ 方法 } & \multicolumn{3}{c}{ 模型 } \\
\cline { 2 - 4 } & 犰狳 & 狗 & 佛像 \\
\hline 文献[5] & 91.210543 & 35.261134 & 102.256187 \\
文献[6] & 93.654221 & 36.233435 & 113.522377 \\
文献[10] & 96.221643 & 34.386741 & 120.207631 \\
文献[11] & 94.277325 & 29.366215 & 114.217223 \\
文献[12] & 87.364275 & 31.412537 & 98.352235 \\
本文 & 86.034954 & 27.338304 & 95.244371 \\
\hline
\end{tabular}

\section{3 本文方法的局限性}

本文的自相似性分析方法仅限于具有点云模 型的物体表面, 其在理论上适用于一般点云, 如城 市场景. 但在扫描过程中, 数据丢失和噪声的影响 可能会影响自相似性分析的精度. 另外，点云模型 点的法向和曲率估计也会引起 SI 和 SDF 的计算误 差, 特别是对于不同尺度但几何相似的部件, 这将 最终影响自相似性分析结果. 本文方法目前依赖
于点云模型的过分割结果, 也会影响到模型的自 相似性分析. 如何减少这些限制, 对物体进行自相 似性分析将是今后的工作.

\section{4 结 语}

本文提出了一种基于特征张量的 $3 \mathrm{D}$ 点云模型 局部自相似性分析方法. 首先对点云模型建立了 点的 GS, SI 和 SDF 的近似计算方法. 将这些特征 组合成特征张量表示模型的每个子块; 然后利用 张量范数及其分量范数进行相似块匹配, 并将其 转化为形状描述符, 用于模型的局部自相似性分 析. 该形状描述符能够充分地结合模型的各种几 何特征, 因此, 形状分析的结果比其他最先进的方 法更可靠.

从理论上讲，基于张量的描述符可以通过考 虑更多的层来集成其他特征来改进. 例如, 如何将 具有自相似性特征的分形方法 ${ }^{[28]}$ 与其他特征描述 符相结合，对一个对象或不同对象的不同块进行 相似性分析，也是今后的工作之一。

\section{参考文献(References):}

[1] Guerrero P, Auzinger T, Wimmer M, et al. Partial shape matching using transformation parameter similarity[J]. Com- 
puter Graphics Forum, 2015, 34(1): 239-252

[2] Kleiman Y, van Kaick O, Sorkine-Hornung O, et al. SHED: shape edit distance for fine-grained shape similarity[J]. ACM Transactions on Graphics, 2015, 34(6): Article No.235

[3] Gal R, Cohen-Or D. Salient geometric features for partial shape matching and similarity[J]. ACM Transactions on Graphics, 2006, 25 (1): 130-150

[4] Arbel N Y, Tal A, Zelnik-Manor L. Partial correspondence of 3D shapes using properties of the nearest-neighbor field[J]. Computers \& Graphics, 2019, 82(8): 183-192

[5] Vock R, Dieckmann A, Ochmann S, et al. Fast template matching and pose estimation in 3D point clouds[J]. Computers \& Graphics, 2019, 79: 36-45

[6] Itskovich A, Tal A. Surface partial matching and application to archaeology[J]. Computers \& Graphics, 2011, 35(2): 334-341

[7] Gatzke T, Grimm C, Garland M, et al. Curvature maps for local shape comparison[J]. International Conference on Shape Modeling and Applications, 2005: 244-253

[8] Koenderink J J, van Doorn A J. Surface shape and curvature scales[J]. Image and Vision Computing, 1992, 10(8): 557-564

[9] Shapira L, Shamir A, Cohen-Or D. Consistent mesh partitioning and skeletonisation using the shape diameter function $[\mathrm{J}]$. The Visual Computer, 2008, 24(4): 249-259

[10] Wang S F, Li N N, Li S, et al. Multi-scale mesh saliency based on low-rank and sparse analysis in shape feature space[J]. Computer Aided Geometric Design, 2015, 35-36: 206-214

[11] Song R, Liu Y H, Martin R R, et al. Mesh saliency via spectral processing[J]. ACM Transactions on Graphics, 2014, 33(1): Article No.6

[12] Wu J L, Shen X Y, Zhu W, et al. Mesh saliency with global rarity[J]. Graphical Models, 2013, 75(5): 255-264

[13] Lee C H, Varshney A, Jacobs D W. Mesh saliency[J]. ACM Transactions on Graphics, 2005, 24(3): 659-666

[14] Bronstein M M, Kokkinos I. Scale-invariant heat kernel signatures for non-rigid shape recognition[C] //Proceedings of the IEEE Conference on Computer Vision and Pattern Recognition. Los Alamitos: IEEE Computer Society Press, 2010: 1704-1711

[15] Du G G, Yin C L, Zhou M Q, et al. Part-in-whole matching of rigid 3D shapes using geodesic disk spectrum[J]. Multimedia Tools and Applications, 2018, 77(15): 18881-18901

[16] Maximo A, Patro R, Varshney A, et al. A robust and rotationally invariant local surface descriptor with applications to non-local mesh processing[J]. Graphical Models, 2011, 73(5):
231-242

[17] Andreopoulos A, Tsotsos J K. 50 years of object recognition: directions forward[J]. Computer Vision and Image Understanding, 2013, 117(8): 827-891

[18] Hu R Z, Fan L B, Liu L G. Co-segmentation of 3D shapes via subspace clustering[J]. Computer Graphics Forum, 2012, 31(5): 1703-1713

[19] Yao B, Li Z, Ding M, et al. Three-dimensional protein model similarity analysis based on salient shape index[J]. BMC Bioinformatics, 2016, 17: Article No.131

[20] Meyer M, Desbrun M, Schröder P, et al. Discrete differential-geometry operators for triangulated 2-manifolds[J]. Mathematics \& Visualization, 2002, 6(8): 35-57

[21] Bradford J R, Westhead D R. Improved prediction of protein-protein binding sites using a support vector machines approach[J]. Bioinformatics, 2005, 21(8): 1487-1494

[22] Zhang Meng, Chen Shuangmin, Shu Zhenyu, et al. The shape diameter function on point clouds[J]. Journal of ComputerAided Design \& Computer Graphics, 2017, 7 (29): 1203-1209 (in Chinese)

(张猛，陈双敏，舒振宇，等. 点云曲面上的形状直径函数 [J]. 计算机辅助设计与图形学学报, 2017, 7 (29): 1203-1209)

[23] Zhang X, Wen G J, Dai W. A tensor decomposition-based anomaly detection algorithm for hyperspectral image[J]. IEEE Transactions on Geoscience and Remote Sensing, 2016, 54(10): 5801-5820

[24] Lu L, Lévy B, Wang W P. Centroidal Voronoi Tessellation of line segments and graphs[J]. Computer Graphics Forum, 2012, 31(2): 775-784

[25] Belkin M, Niyogi P. Laplacian eigenmaps for dimensionality reduction and data representation[J]. Neural Computation, 2003, 15(6): 1373-1396

[26] Huang H, Wu S H, Cohen-Or D, et al. $L_{1}$-medial skeleton of point cloud[J]. ACM Transactions on Graphics, 2013, 32 (4): Article No.65

[27] Daszykowski M, Kaczmarek K, Vander H Y, et al. Robust statistics in data analysis-a review: basic concepts[J]. Chemometrics and Intelligent Laboratory Systems, 2007, 85(2): 203-219

[28] Hu H L, Li Z, Dong H W, et al. Graphical representation and similarity analysis of protein sequences based on fractal interpolation[J]. IEEE/ACM Transactions on Computational Biology and Bioinformatics, 2017, 14(1): 182-192 